\title{
Expression of Gjal correlates with the phenotype observed in oculodentodigital syndrome/type III syndactyly
}

\author{
R R Richardson, D Donnai, F Meire, M J Dixon
}

J Med Genet 2004;41:60-67

O culodentodigital syndrome (ODD; OMIM 164200) is a congenital disorder characterised by developmental abnormalities of the face, eyes, limbs, and dentition. ODD is inherited in an autosomal dominant fashion and displays high penetrance but variable expression. ${ }^{1}$ In addition, a high rate of de novo mutations is observed. ${ }^{2}$ Facially, affected patients exhibit a long, narrow nose with hypoplastic alae, thin, anteverted nostrils and a prominent nasal bridge, short palpebral fissures, and bilateral microcornea often with anomalies of the iris. ${ }^{3}{ }^{4}$ Secondary glaucoma occurs in a number of patients. ${ }^{5}$ Bilateral complete syndactyly of the fourth and fifth fingers (type III syndactyly) is the characteristic digital malformation. The third finger may occasionally also be involved and associated camptodactyly is a common finding. ${ }^{2}$ In addition, microdontia and generalised hypoplasia of the enamel, which tends to affect both the primary and secondary dentitions, are frequently observed. ${ }^{2}{ }^{6}$ Cleft palate has also been reported in a number of cases. ${ }^{6-9}$ Less common features include thin, sparse hair and conductive deafness. Spastic paraparesis or lower limb weakness in association with ODD has been reported in a number of sporadic and familial cases. ${ }^{269-11}$ In two of these reports, magnetic resonance imaging demonstrated an underlying leukodystrophy and it has, therefore, been proposed that the definition of ODD be widened to include these features. ${ }^{10} 11$ Type III syndactyly has also been reported to occur as an isolated entity in several autosomal dominant pedigrees and it is uncertain whether this anomaly and ODD are separate genetic entities or part of the same disease spectrum. ${ }^{12-15}$ However, a family has been reported who, while not exhibiting the usual ocular or dental anomalies associated with ODD, did have a facial appearance that appeared to bridge the gap between the two conditions. ${ }^{16}$

The ODD locus was initially mapped to a $28 \mathrm{cM}$ region of human chromosome 6q22-q24, ${ }^{17}$ which was subsequently refined to the $1.9 \mathrm{cM}$ genetic interval delineated by the polymorphic markers D6S261, proximally, and D6S1639, distally. ${ }^{18}$ This study further indicated that ODD with associated neurological defects maps to this same interval. Recently, Paznekas and coworkers reported that the pleiotropic phenotype of ODD arises as the result of mutation of $G J A 1$, the gene encoding the gap junction protein, connexin $43 .{ }^{19}$ In the current paper, we report nine different missense mutations, seven of which have not been described previously, in 10 unrelated families and confirm that type III syndactyly, at least in a subset of cases, is allelic with ODD. Using whole mount in situ hybridisation to analyse a developmental series of morphologically-staged embryos, we further demonstrate that there is a strong correlation between the sites of Gjal expression and the clinical phenotype.

\section{PATIENTS, MATERIALS AND METHODS \\ Families}

The pedigrees of six of the families analysed in the current study have been presented previously. ${ }^{17}$ The remaining five

\section{Key points}

- Oculodentodigital syndrome (ODD) is an autosomal dominant disorder characterised by developmental abnormalities of the face, eyes, limbs, and dentition. Mutations in GJA1, which encodes the gap junction protein connexin 43, have been shown to underlie ODD.

- Here, we describe an additional 10 mutations in GJA1, seven of which are novel, bringing the total reported to date to 24 . All but one of these mutations result in the introduction of a missense change into the amino terminal two thirds of connexin 43 highlighting the functional importance of this region of the protein. One of these mutations occurs in a family that exhibits type III syndactyly but not the usual ophthalmic, skeletal, or dental findings commonly associated with ODD.

- Analysis of a developmental series of morphologically staged mouse embryos using whole mount in situ hybridisation allowed us to demonstrate a strong correlation between the spatio-temporal expression pattern of Gjal in the developing craniofacial complex and limbs and the pleiotropic features of ODD.

families were referred by clinical geneticists and ophthalmologists after a diagnosis of ODD had been made.

\section{Mutation analysis}

GJAl contains two exons, the coding sequence being encompassed in its entirety by the second exon (GenBank accession numbers: cDNA, M65188; genomic DNA, AL139098). The coding sequence of GJAl was amplified in two overlapping segments using the primers 5'-AAT ACG TGA AAC CGT TGG TAG-3' and 5'-CTC TTT CCC TTA ACC CGA TC-3', which amplified a product of $855 \mathrm{bp}$, and $5^{\prime}$-TCT TTG AGG TGG CCT TCT TG-3' and 5'-TAA GGC TGT TGA GTA CCA CC-3', which amplified a product of $773 \mathrm{bp}$. The PCR amplifications were performed in $25 \mu \mathrm{l}$ volumes containing 50 pmol of each primer, $200 \mu \mathrm{M}$ dNTPs, $10 \mathrm{mM}$ Tris- $\mathrm{HCl}$ $\mathrm{pH}$ 8.3, $50 \mathrm{mM} \mathrm{KCl}, 1.0 \mathrm{mM} \mathrm{MgCl}_{2}$ and $0.01 \%$ gelatin. The samples were overlaid with mineral oil, heated to $96^{\circ} \mathrm{C}$ for $10 \mathrm{~min}$ and cooled to $55^{\circ} \mathrm{C}$. After addition of $0.75 \mathrm{U}$ Taq DNA polymerase, the samples were processed through 35 amplification cycles of $92^{\circ} \mathrm{C}$ for $60 \mathrm{~s}, 55^{\circ} \mathrm{C}$ for $60 \mathrm{~s}$, and $72^{\circ} \mathrm{C}$ for $60 \mathrm{~s}$ using a Hybaid thermal cycler. The final extension step was lengthened to $10 \mathrm{~min}$. Negative controls were established for all reactions. After amplification, the products were excised from a $1 \%$ agarose gel and sequenced directly using the

Abbreviations: ODD, oculodentodigital syndrome 
dideoxy chain termination method and dye primer chemistry. Primers were designed to avoid amplification of the processed pseudogene GJA1P1 on human chromosome 5q21-q22.

\section{Whole mount in situ hybridisation}

Sense and anti-sense riboprobes were generated from a Gjal cDNA clone (IMAGE clone 1228506) that encompassed the last $390 \mathrm{bp}$ of coding sequence plus $1726 \mathrm{bp}$ of the 3' untranslated region using the $\mathrm{T} 3$ and $\mathrm{T} 7$ promoters, respectively. Embryos dissected from time mated MF1 mice were fixed in $4 \%$ paraformaldehyde overnight, processed and subjected to hybridisation with sense and anti-sense probes as described previously ${ }^{20}$ with the modifications that hybridisations were performed at $60^{\circ} \mathrm{C}$ for $3-4$ days and the detection reaction was performed at $4^{\circ} \mathrm{C}$.

\section{RESULTS}

Mutation analysis

Analysis of the entire GJAl coding sequence and the associated splice junctions resulted in the identification of nine different mutations, seven of which have not been reported previously, in the affected members of 10 out of the 11 ODD families (fig 1, table 1). No mutations were found in DNA samples from the two affected individuals from the remaining family. In each case the mutation segregated with the disease phenotype and was not present in 100 normal chromosomes. Each mutation resulted in the introduction of a missense change into connexin 43 and all of the mutated amino acid residues are highly conserved across connexin 43 orthologues (fig 2). The mutations are spread throughout the protein with three falling in the first transmembrane domain (nt79T $>C$ resulting in $\mathrm{S} 27 \mathrm{P}$; $\mathrm{nt} 93 \mathrm{~T}>\mathrm{G}$ resulting in $\mathrm{I} 31 \mathrm{M}$; nt119C $>\mathrm{T}$ resulting in $\mathrm{A} 40 \mathrm{~V})$, one in the first extracellular loop (nt206C $>$ A resulting in S69Y), four in the cytoplasmic loop (nt338T $>C$ resulting in L113P; $n t 402 \mathrm{G}>\mathrm{T}$ resulting in K134N; nt427G $>$ A resulting in G143S; nt443G $>$ A resulting in $\mathrm{R} 148 \mathrm{Q})$, and one in the second extracellular loop (nt605G $>$ A resulting in $\mathrm{R} 202 \mathrm{H}$ ) (fig 2). The mutation G143S is of particular interest as it underlies the atypical phenotype of the family reported by Brueton and coworkers, members of which display type III syndactyly but none of the usual ophthalmological, dental or skeletal features commonly reported in ODD. ${ }^{16}$ The mutations $\mathrm{A} 40 \mathrm{~V}$ and $\mathrm{R} 202 \mathrm{H}$ have been reported previously. ${ }^{19}$ A mutation of the lysine residue at position 134 has also been reported previously, ${ }^{19}$ however, the mutation identified in the current study is different as it results in a change to asparagine rather than glutamic acid. Interestingly, two of the mutations that we have identified (nt119C $>$ T resulting in $\mathrm{A} 40 \mathrm{~V}$ and $\mathrm{nt} 443 \mathrm{G}>\mathrm{A}$ resulting in R148Q) result in a change to a nucleotide that is present in the GJAl pseudogene (GJAIPI) located on human chromosome 5q21-q22, which shares 97\% identity with GJAl. However, we can exclude the possibility that we have inadvertently amplified GJAIPI as none of the other nucleotides that define the GJAIPI sequence were detected in our amplification products. Interestingly, one of these mutations, R148Q, was detected in two unrelated families.

\section{Expression analysis}

To correlate the expression of Gjal during embryogenesis with the phenotypic features of ODD, a developmental series of morphologically staged mouse embryos was analysed using whole mount in situ hybridisation.

\section{Expression in the craniofacial complex}

Strong expression of Gjal was detected throughout the craniofacial region at E8.5; this was particularly marked in regions anterior to the pre-otic sulcus at the rhombomere $2 / 3$ boundary, in the most anterior portion of the forebrain surrounding the optic pits, and in the developing neural folds (figs 3A, 3B). Strong expression was also observed in the caudal neuropore. At E9.5, expression of Gjal was detected in the frontonasal process and the developing branchial arches. In particular, the maxillary and mandibular portions of the first branchial arch and the second branchial arch exhibited strong expression (figs 3C, 3D). At E10.5, a gradient in the expression domain of Gjal was evident in the developing mandible with the highest levels concentrated towards the midline (fig 3F). In addition, domains of Gjal expression, very similar to those of Bmp $4,{ }^{21}$ were detected in the medial and lateral nasal processes, at the most proximal regions surrounding the nasal pit (fig 3E). Gjal expression was also detected in the developing eye. At E12.5, expression was detected in the developing snout, particularly in the hair follicles and the primordia of the vibrissae. At E13.5, Gjal expression was detected in the incisor and molar tooth germs. Although Gjal expression was not detected in the palatal shelves prior to their elevation (E13), strong expression was detected as they underwent fusion at E14.5 (figs 3G, 3H). Gjal expression was not, however, detected after completion of palatal fusion on E15 (data not shown).

\section{Expression of Gial in the developing limb}

At E9.5, strong expression of Gjal was detected in the limb bud and the somites (fig 4A). At E10.5, a broad expression domain was present in the limb bud, however, by E11.5, this domain became localised predominantly to the apical ectodermal ridge (figs $4 \mathrm{~B}, 4 \mathrm{C}$ ). No obvious gradients in the expression of Gjal along the anterior-posterior axis of the limb bud were observed. From E12.5, the expression within the apical ectodermal ridge persisted but areas of reduced expression were apparent in the interdigital regions (fig 4D). At E13.5, Gjal expression was localised to the surface ectoderm of the limbs, in regions corresponding to the future digits (fig 4E). Strong expression domains were observed at the most distal portions of the digits. At E14.5 and E15.5, Gjal expression was present in the dorsal and ventral surfaces of the digits and the hair follicles in the wrist (figs $4 \mathrm{~F}, 4 \mathrm{G}$ ).

This wide ranging expression pattern of Gjal correlates with the pleiotropic phenotype observed in patients with ODD as the main areas of strong expression are in the eye, limbs, dentition, nasal region, fusing secondary palate, and hair follicles.

\section{DISCUSSION}

Connexin 43, encoded by GJAl, belongs to a family of transmembrane proteins each of which is composed of intracytoplasmic amino and carboxy termini and four membrane spanning domains linked by two extracellular and one intracellular loop. Six connexin proteins form a hemichannel or connexon which spans the cell membrane allowing connexons from neighbouring cells to dock to form a complete channel or gap junction. Gap junctions allow the exchange of secondary messengers, ions, and other small molecules up to the size of $1 \mathrm{kDa}$ between adjacent cells thereby forming a system of cell-cell communication that operates alongside ligand-receptor signalling. Gap junction communication plays a crucial role not only in normal tissue physiology but in the regulation of information flow required during embryonic morphogenesis. ${ }^{22}$ Mutations in the genes encoding various connexins have been demonstrated to underlie a wide range of genetic disorders including Charcot-Marie-Tooth syndrome, deafness, dermatological disorders, cataracts and heart malformations, suggesting a wide range of expression patterns and functional diversity. ${ }^{23-25}$ 

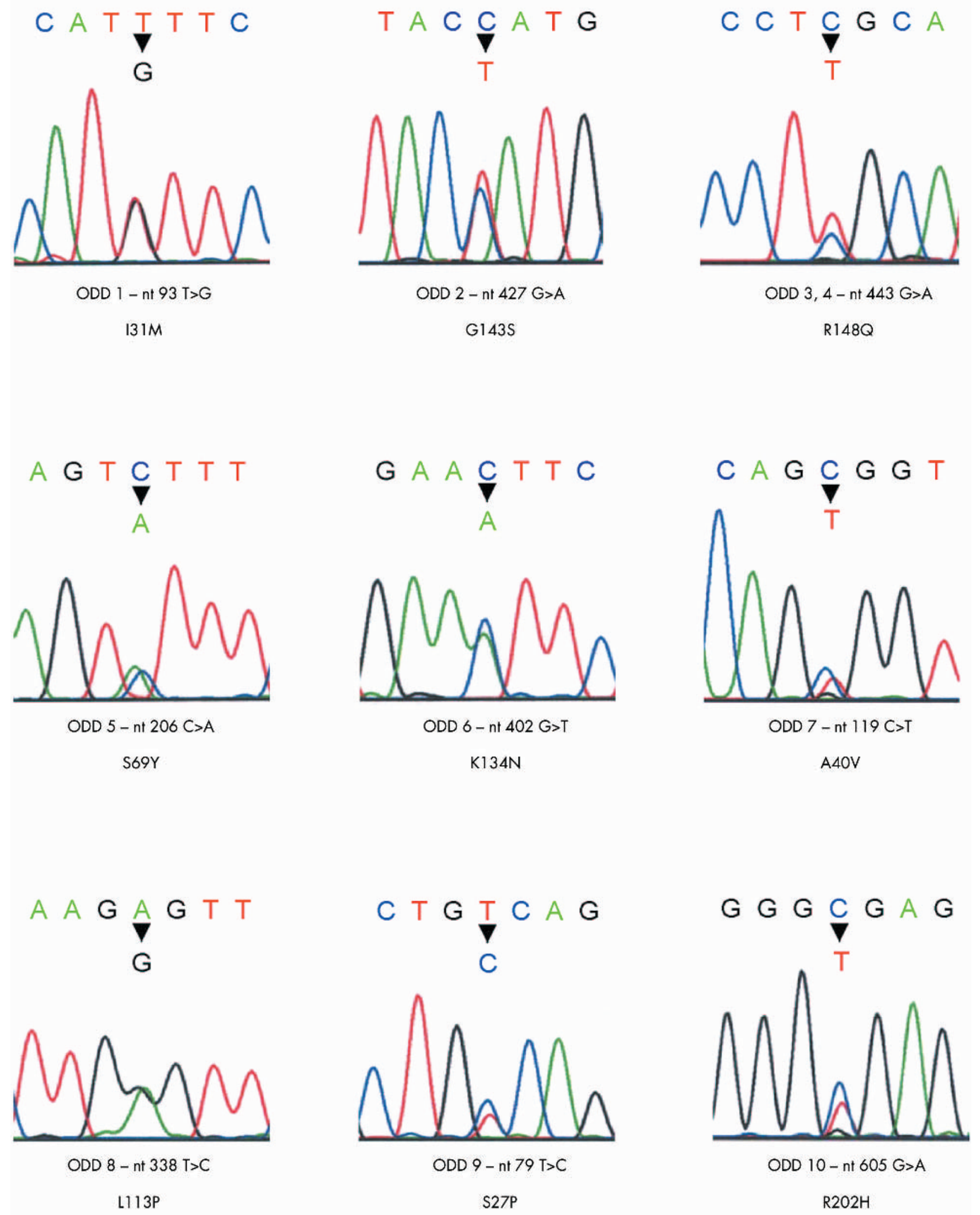

Figure 1 Mutation analysis in ODD. The nine different heterozygous mutations detected in GJAl are depicted. In the case of families ODD1, ODD5, ODD7, and ODD9, the coding strand is shown, the non-coding strand being presented in the remaining cases.

Recently, Paznekas and coworkers showed that mutations in GJAl underlie the congenital malformation oculodentodigital syndrome. ${ }^{19}$ In the current investigation, we have extended these findings by delineating a further nine GJAl mutations, seven of which are novel, in 10 ODD families bringing the total number of different mutations reported to 
Table 1 Mutations identified in GJAl

\begin{tabular}{|c|c|c|c|}
\hline Family number & Mutation in GJAl & Mutation in connexin 43 & Position of mutation in connexin 43 \\
\hline ODDI & $n+93 T>G$ & $131 \mathrm{M}$ & Transmembrane domain 1 \\
\hline ODD2 & $n+427 G>A$ & G143S & Cytoplasmic loop \\
\hline ODD3 & $n+443 G>A$ & R148Q & Cytoplasmic loop \\
\hline ODD4 & $n+443 G>A$ & R148Q & Cytoplasmic loop \\
\hline ODD5 & $n+206 C>A$ & S69Y & Extracellular loop 1 \\
\hline ODD6 & $n+402 G>T$ & $\mathrm{~K} 134 \mathrm{~N}$ & Cytoplasmic loop \\
\hline ODD7 & $n+119 C>T$ & A40V & Transmembrane domain 1 \\
\hline ODD8 & $n+338 \mathrm{~T}>\mathrm{C}$ & L113P & Cytoplasmic loop \\
\hline ODD9 & nt79T $>C$ & S27P & Transmembrane domain 1 \\
\hline ODD10 & $n+605 G>A$ & $\mathrm{R} 2 \mathrm{O} 2 \mathrm{H}$ & Extracellular loop 2 \\
\hline
\end{tabular}

date to 24. One of these mutations was found to underlie the atypical phenotype of the family reported by Brueton and coworkers ${ }^{16}$ and our observations therefore indicate that type III syndactyly, at least in this family, is allelic with typical ODD. All but one of the mutations that have been reported to date lead to missense changes in connexin 43 , the remaining mutation being a duplication of a phenylalanine residue at position 52. In all cases, the mutations occur in amino acid residues that are highly conserved during evolution (fig 2). The lack of mutations resulting in the introduction of a termination codon into the protein suggests that the mechanism underlying ODD is not a loss of connexin 43 function. This hypothesis is reinforced by the observation that nullizygosity of Gjal in mice does not lead to an ODDlike phenotype. Rather, mice lacking connexin 43 died at birth, as a result of a failure in pulmonary gas exchange caused by swelling and blockage of the right ventricular outflow tract from the heart. ${ }^{26}$ These observations suggest that a dominant negative or "gain of function" mechanism might underlie ODD.

The 24 different mutations reported to underlie ODD are distributed throughout the different functional domains of the first two thirds of connexin 43 (fig 2). Most of the 5' mutations that have been described fall within the 19 amino acid N-terminal domain of connexin 43 (Y17S, S18P, ${ }^{19}$ ), a region that has been shown to be involved in voltage gating of gap junction channels in connexins 26 and $32 .{ }^{27}{ }^{28}$ Eight of the mutations that have been described to date (G21R, G22E, K23T, S27P, I31M, A40V, L90V, V216L) occur in the transmembrane domains of connexin 43, the integrity of
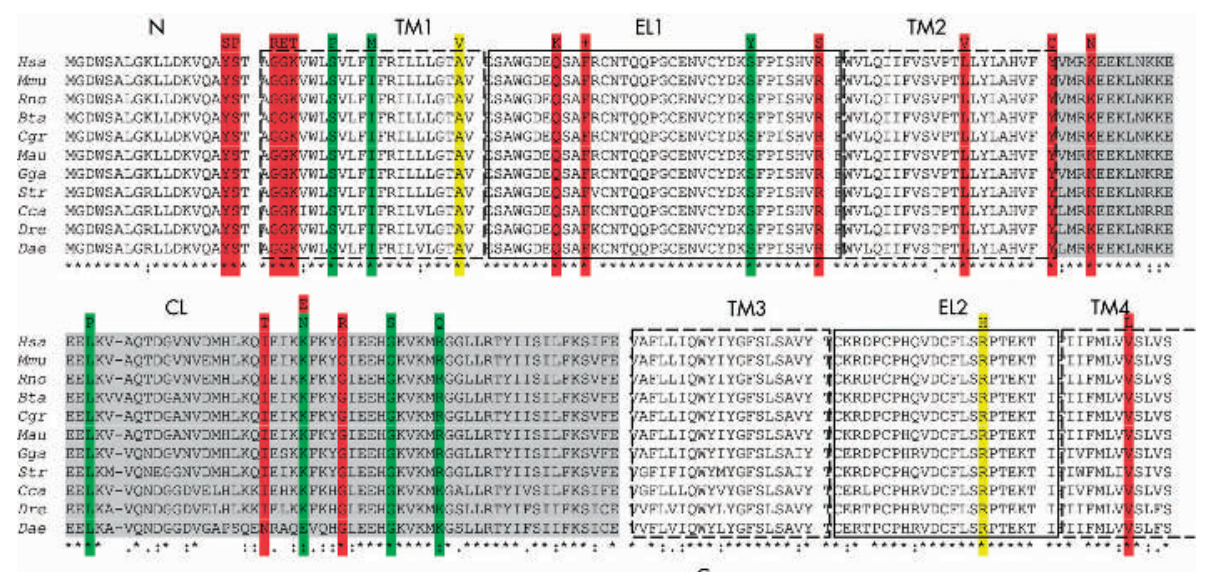

C

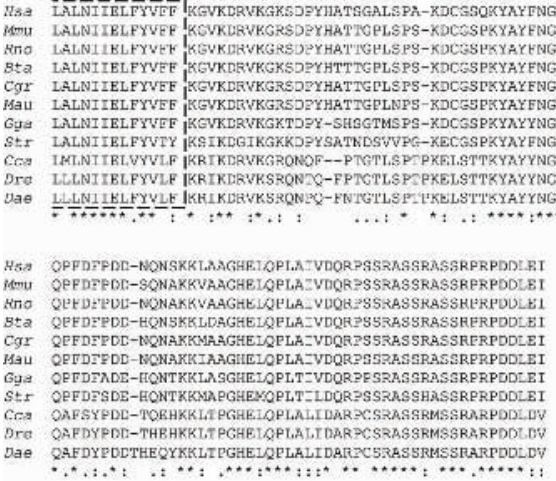

Figure 2 Alignment of connexin 43 orthologues. Human ( $H s a)$, mouse (Mmu), rat (Rno), cow (Bta), Chinese hamster (Cgr), golden hamster (Mau), chicken (Gga), western clawed frog (Str), carp (Cca), zebrafish (Dra), and Danio aequipinnatus (Dae) amino acid sequences have been aligned using CLUSATLW. The mutations in connexin 43 that underlie ODD are indicated above the alignment. Mutations highlighted in green are those identified in the current study, mutations highlighted in red are those described by Paznekas and coworkers (2003) and mutations highlighted in yellow have been observed in both studies. The four transmembrane domains (TM1, TM2, TM3, and TM4) are outlined with dashed boxes and the two extracellular loops (EL1, EL2) with solid boxes. The cytoplasmic loop (CL) is shaded grey and the amino terminal (N) and carboxy terminal (C) domains are unshaded. 

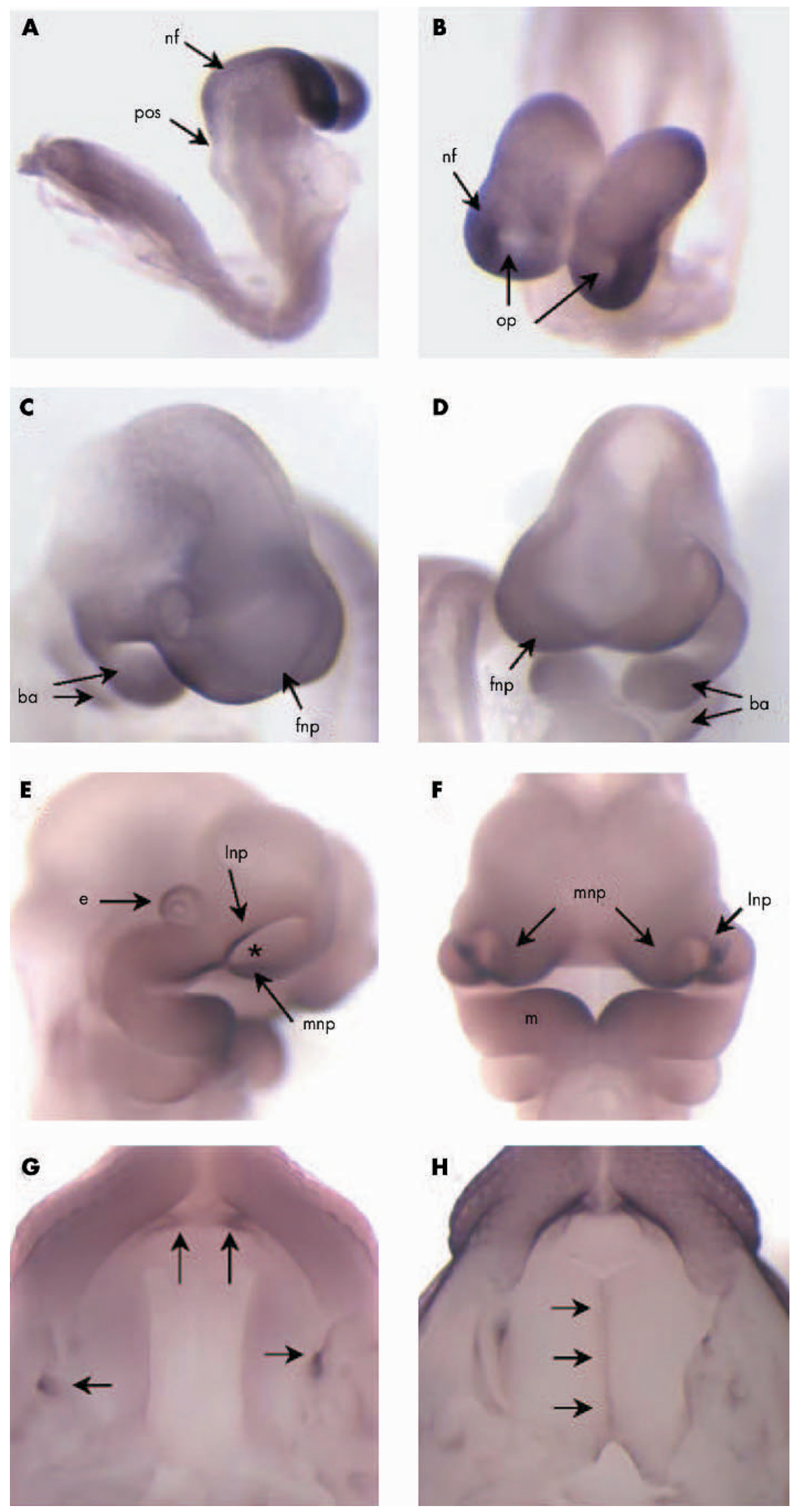

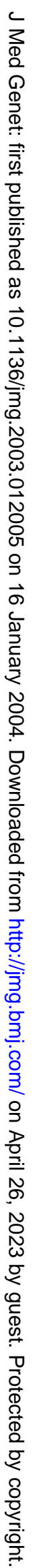



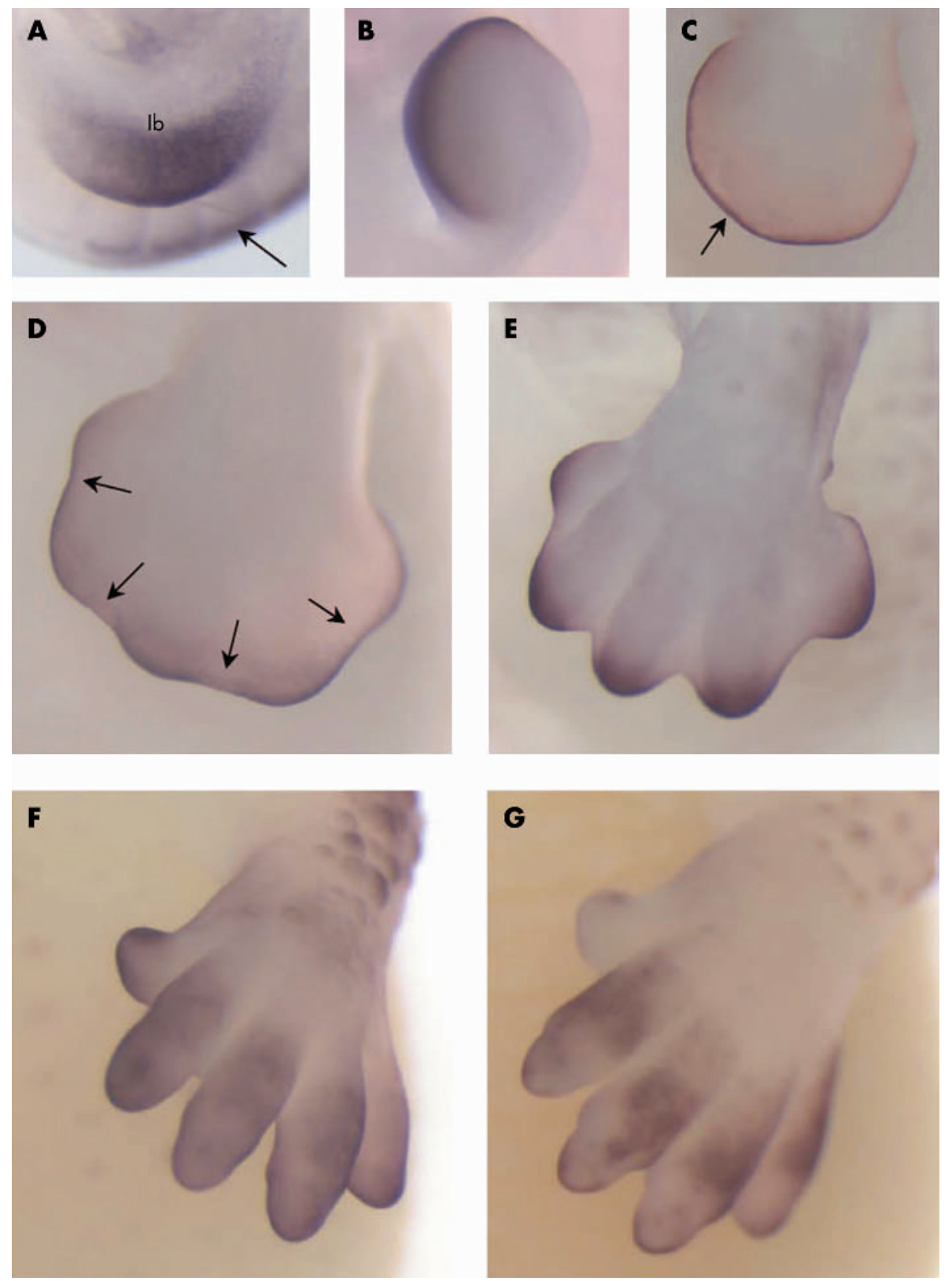

Figure 4 Expression of Gjal in the developing limb as analysed by whole mount in situ hybridisation. (A) At E9.5, intense expression of Gial was detected in the limb bud (lb) and the somites (arrowed). (B) At E10.5, a broad and diffuse domain of Gial expression was present throughout the limb bud, however, by E1 1.5 (C), this domain became localised to the apical ectodermal ridge (arrowed). (D) At E12.5, expression of Gjal was localised to the most distal portions of the limb. Areas of reduced expression were distinct in the future interdigital regions (arrowed). (E) At E1 3.5, Gjal expression was localised to the surface ectoderm of the limbs, in regions corresponding to the future digits. Expression was particularly intense at the distal portion of the digit. At E14.5 (F) and E15.5 (G), Gial expression was present in the dorsal and ventral surfaces of the digits and the hair follicles in the wrist.

which is known to be essential for correct transport of the protein into the plasma membrane. ${ }^{29}$ While the precise contribution of each domain to the formation of the pore of the gap junction, and therefore channel permeability, remains controversial, the first transmembrane domain has been strongly implicated in this process. ${ }^{30} 31$ It is therefore interesting that six of the transmembrane mutations identified in ODD families fall within this region of connexin 43. In total, five mutations (Q49K, F52dup, S69Y, R76S, $\mathrm{R} 202 \mathrm{H}$ ) have now been identified in the two extracellular

Figure 3 (From previous page) Expression patterns of Gjal in the developing craniofacial complex as analysed by whole mount in situ hybridisation. Lateral (A) and dorsal (B) views of an E8.5 embryo indicate that strong expression of Gjal was detected throughout the craniofacial region at E8.5. This was particularly marked anterior to the pre-otic sulcus (pos), in the forebrain surrounding the optic pits (op), and in the developing neural folds (nf). (C, D) AtE9.5, Gjal expression was detected in the frontonasal process (fnp) and the developing branchial arches (ba). Lateral (E) and frontal (F) views of an E10.5 embryo reveal strong Gjal expression in the developing eye (e; arrowed) and in the medial and lateral nasal processes (mnp and lnp, respectively), which was particularly intense in the most posterior regions surrounding the nasal pit (asterisked). A gradient of Gjal expression was observed in the mandibular processes $(\mathrm{m})$, the highest levels of expression being observed towards the midline. (G) Expression of Gjal at E13.5. Intense domains of Gjal expression were detected in the incisor and molar tooth germs (arrowed). (H) Gjal expression was detected in the shelves of the secondary palate as they underwent fusion at E14.5 (arrowed). 
loops of connexin 43. Mutations in this region of connexin 43 might interfere with relative spacing of the cysteine residues in the extracellular loops, which is critical for appropriate disulphide bond formation and the specific docking of two hemichannel connexons to form a functional gap junction. ${ }^{32}$ In this context, Paznekas and coworkers ${ }^{19}$ reported the mutation Q49K in one ODD family. Mutation of the glutamine residue at position 49 to histidine in chick connexin 43 did not allow the production of connexin 43 channels, most probably through the distortion of the secondary structure of the first extracellular loop. ${ }^{33}$ Alternatively, the mutations may interfere with the voltage gating of the entire channel. The remaining nine mutations that have been identified in GJAl affect the cytoplasmic loop of connexin 43. The amino acid composition and size of this region of the connexin family is highly diverse, nevertheless, all of the mutations that have been identified in this region of connexin 43 affect highly conserved residues (fig 2). Moreover, antibodies directed against amino acids 123-136 and 131-142 of connexin 43, which encompass I130, K134 and G138 that are mutated in ODD, prevented intercellular communication through mouse embryo gap junctions. ${ }^{34}$ In summary, the consequence of any of the 24 mutations found in connexin 43 to date may be to disrupt the docking of the connexins to form a hemichannel or the two hemichannels to form an entire gap junction, or to affect the permeability of the fully formed gap junction. Interestingly, no ODD mutations have, as yet, been identified in the 149 amino acid carboxy terminal cytoplasmic domain. This distribution suggests that mutations in this region of connexin 43 might lead to a different phenotype. In this context, the S365P mutation is thought to result in autosomal recessive cardiac malformations with laterality defects in a subset of cases. ${ }^{35}$

The strong correlation between the sites of Gjal expression during mouse embryonic development and the ODD phenotype provide further support for mutations in GJAI underlying ODD. The earliest stages examined in the current study (E8) allowed us to demonstrate that Gjal is expressed in the most anterior portion of the forebrain and in the developing neural folds. These results confirm the earlier report of Lo and coworkers $^{36}$ and are consistent with the proposed role of connexin 43 in the modulation of neural crest cell motility. ${ }^{37}$ Similarly, the Gjal expression patterns that we observed in the branchial arches and developing facial processes mirror those reported for connexin 43 in the chick. ${ }^{38}$ The highest levels of chicken connexin 43 expression were concentrated in the mesenchyme at the distal edges of the medial nasal, lateral nasal and maxillary processes suggesting a role in fusion of the facial primordia. ${ }^{38}$ In this context, application of antisense oligonucleotides that specifically reduced the levels of connexin 43 protein in cells of the early chick facial primordia resulted in craniofacial anomalies consistent with those observed in ODD. ${ }^{39}{ }^{40}$ Our observation that Gjal is also specifically expressed in the medial edges of the paired palatal shelves immediately before and during fusion of the murine secondary palate is interesting because of the cleft palate that has been reported in a number of patients with ODD. This expression pattern implies a key role for connexin 43 in the adhesion or fusion of the palatal shelves. The process of epithelial-mesenchymal transformation has been implicated in palatal shelf fusion ${ }^{41}{ }^{42}$ and, in this context, it is perhaps significant that gap junction communication is an important regulator of epithelial-mesenchyma transformation during heart development. ${ }^{43}$ Additional craniofacial expression of Gjal was detected in the developing eyes, vibrissae, and molar and incisor tooth germs mirroring the ocular anomalies, abnormal hair growth, and microdontia/ enamel hypoplasia observed in patients with ODD. These results are also consistent with the reported expression of connexin 43 in rat incisor odontoblasts, ${ }^{44}{ }^{45}$ human dental pulp fibroblasts ${ }^{46}$ and during ocular development in Xenopus, mouse and chick. ${ }^{47-49}$

Finally, we have shown that Gjal is expressed across the entire limb bud at E9, but, by E10, Gjal is restricted to the AER. Meyer and coworkers have previously shown that Gjal is dynamically expressed during early limb development in the mouse and that ectopic expression of Wntl in the limb mesenchyme leads to alterations in Gjal expression in conjunction with limb abnormalities. ${ }^{50}$ Similarly, Gjal is expressed during limb development in Xenopus and chick $^{39} 48$ and knockdown of Gjal in chicks leads to limb anomalies. ${ }^{39}$ Later in limb development, expression of Gjal is progressively down-regulated in the interdigital webs but retained in the digits themselves. As with the craniofacial expression of Gjal, these results are consistent with the syndactyly observed in ODD.

Taken together, our results indicate that connexin 43 plays a key role in normal facial and limb development and that mutation of GJAl results in craniofacial anomalies and limb abnormalities. Nevertheless, the precise way in which mutant connexin 43 disrupts gap junction communication and leads to the malformations observed in ODD remains to be elucidated.

\section{ACKNOWLEDGEMENTS}

We thank the families for participating in the study and A Aylsworth, L Brueton, S Farrell, A Fryer, R Hennekam, K Metcalfe, A Murray, C Schrander-Stumpel, H Toriello, A Verloes, and R Winter for providing samples.

We should also like to thank E De Baere and L Messiaen for critical appraisal of the manuscript.

\section{Authors' affiliations}

R Richardson, M J Dixon, School of Biological Sciences and Department of Dental Medicine and Surgery, University of Manchester, Manchester, M13 9PT, UK

D Donnai, Academic Unit of Medical Genetics and Regional Genetic Service, St Mary's Hospital, Manchester, MI3 OJH, UK

F Meire, Department of Paediatric Ophthalmology, University of Gent, De Pintelaan 185, B-9000 Ghent, Belgium

This work was supported by grants from the Wellcome Trust (058423, 069243).

Correspondence to: Professor M J Dixon, School of Biological Sciences and Department of Dental Medicine and Surgery, 3.239 Stopford Building, University of Manchester, Oxford Road, Manchester, M1 3 9PT, UK; mike.dixon@man.ac.uk

Received 3 July 2003

Accepted 31 July 2003

\section{REFERENCES}

1 Judisch GF, Martin-Casals A, Hanson JW et al. Oculodentodigital dysplasia: four new reports and a literature review. Arch Ophthalmol 1979;97:878-84.

2 Reisner SH, Kott E, Bornstein B, et al. Oculodentodigital dysplasia. Am J Dis Child 1969:118:600-7.

3 Sugar HS, Thompson JP, Davis JD. The oculodento-digital dysplasia syndrome. Am J Ophthalmol 1966;61:1448-51.

4 Dudgeon J, Chisholm IA. Oculodentodigital dysplasia. Trans Ophthalmol Soc U K 1974;94:203-10.

5 Sugar HS. Oculodentodigital dysplasia syndrome with angle-closure glaucoma. Am J Ophthalmol 1978;86:36-8.

6 Fara M, Horak I, Hrivnakova J, et al. Oculodentodigital dysplasia. Acta Chir Plast 1977; 19:110-22.

7 Gillespie FD. Hereditary dysplasia oculodentodigitalis. Arch Ophthalmol 1964;71:187-92

8 Weintraub DM, Baum JL, Pashayan HM. A family with oculodentodigital dysplasia. Cleft Palate J 1975;12:323-9.

9 Nivelon-Chevallier A, Audry D, Audry F, et al. Dysplasie oculo-dento-digitale. A propos d'un cas avec paraplegie spasmodique. J Genet Hum 1981;29:171-9.

10 Gutmann DH, Zackai EH, McDonald-McGinn DM, et al. Oculodentodigital dysplasia syndrome associated with abnormal cerebral white matter. Am J Med Genet 1990;41:18-20. 
11 Norton KK, Carey JC, Gutmann DH. Oculodentodigital dysplasia with cerebral white matter abnormalities in a two-generation family. Am J Med Genet 1995;57:458-61.

12 Bell J. Three further cases of hereditary digital anomaly seen in the out-patient department of Great Ormond Street Hospital for Sick Children. Ann Eugen $1931 ; 4: 233-7$.

13 Collette AT. A case of syndactylism of the ring and little fingers. Am J Hum Genet 1954;6:241-3.

14 Johnston O, Kirby WV. Syndactyly of the ring and little finger. Am J Hum Genet 1955;7:80-2

15 Temtamy SA, McKusick VA. The genetics of hand malformations. Birth Defects 1978;14:309-14

16 Brueton LA, Huson SM, Farren B, et al. Oculodentodigital dysplasia and type III syndactyly: separate genetic entities or disease spectrum? J Med Genet 1990;27:169-75.

17 Gladwin AJ, Donnai D, Metcalfe K, et al. Localization of a gene for oculodentodigital syndrome to human chromosome 6q22-q24. Hum Mol Genet 1997;6:123-7.

18 Boyadjiev SA, Jabs EW, LaBuda M, et al. Linkage analysis narrows the critical region for oculodentodigital dysplasia to chromosome 6q22-q23. Genomics 1999;58:34-40.

19 Paznekas WA, Boyadjiev SA, Shapiro RE, et al. Connexin 43 (GJA1) mutations cause the pleiotropic phenotype of oculodentodigital dysplasia. Am J Hum Genet 2003;72:408-18.

20 Nieto MA, Patel K, Wilkinson DG. In situ hybridization analysis of chick embryos in whole mount and tissue sections. Methods Cell Biol 1996;51:219-35.

21 Gong SG, Guo C. Bmp4 gene is expressed at the putative site of fusion in the midfacial region. Differentiation 2003;71:228-36.

22 Levin M. Isolation and community: a review of the role of gap-junctional communication in embryonic patterning. J Membr Biol 2001;185:177-92.

23 Krutovskikh V, Yamasaki $\mathrm{H}$. Connexin gene mutations in human genetic diseases. Mutat Res 2000;462:197-207.

24 Kelsell DP, Dunlop J, Hodgins MB. Human diseases: clues to cracking the connexin code. Trends Cell Biol 2001;11:2-6.

25 Rabionet R, Lopez-Bigas N, Arbones ML, et al. Connexin mutations in hearing loss, dermatological and neurological disorders. Trends Mol Med 2002;8:205-12.

26 Reaume AG, de Sousa PA, Kulkarni S, et al. Cardiac malformation in neonatal mice lacking connexin43. Science 1995;267:1831-4.

27 Rubin JB, Verselis VK, Bennett MV, et al. A domain substitution procedure and its use to analyze voltage dependence of homotypic gap junctions formed by connexins 26 and 32. Proc Natl Acad Sci U S A 1992;89:3820-4.

28 Verselis VK, Ginter CS, Bargiello TA. Opposite voltage gating polarities of two closely related connexins. Nature 1994;368:348-51.

29 Leube RE. The topogenic fate of the polytopic transmembrane proteins, synaptophysin and connexin, is determined by their membrane-spanning domains. J Cell Sci 1995; 108:883-94.

30 Zhou XW, Pfahnl A, Werner R, et al. Identification of a pore lining segment in gap junction hemichannels. Biophys J 1997;72:1946-53.

31 Pfahnl A, Dahl G. Gating of cx46 gap junction hemichannels by calcium and voltage. Pflugers Arch 1999;437:345-53.

32 Foote $\mathrm{Cl}$, Zhou L, Zhu X, et al. The pattern of disulfide linkages in the extracellular loop regions of connexin 32 suggests a model for the docking interface of gap junctions. J Cell Biol 1998;140:1187-97.
33 Sokolova IV, Martinez AM, Fletcher WH. The highly conserved Gln49 and Ser50 of mammalian connexin43 are present in chick connexin 43 and essential for functional gap junction channels. Cell Commun Adhes 2002:9.75-86

34 Becker DL, Evans WH, Green CR, et al. Functional analysis of amino acid sequences in connexin 43 involved in intercellular communication through gap junctions. J Cell Sci 1995;108:1455-67.

35 Britz-Cunningham SH, Shah MM, Zuppan CW, et al. Mutations of the connexin 43 gap-junction gene in patients with heart malformations and defects of laterality. N Engl J Med 1995;332:1323-9.

36 Lo CW, Cohen MF, Huang GY, et al. Cx43 gap junction gene expression and gap junctional communication in mouse neural crest cells. Dev Genet 1997;20:119-32.

37 Xu X, Li WE, Huang GY, et al. Modulation of mouse neural crest cell motility by $\mathrm{N}$-cadherin and connexin 43 gap junctions. J Cell Biol 2001;154:217-30.

38 Minkoff R, Parker SB, Rundus VR, et al. Expression patterns of connexin 43 protein during facial development in the chick embryo: associates with outgrowth, attachment, and closure of the midfacial primordia. Anat Rec 1997;248:279-90.

39 Becker DL, McGonnell I, Makarenkova HP, et al. Roles for alpha 1 connexin in morphogenesis of chick embryos revealed using a novel antisense approach. Dev Genet 1999;24:33-42.

40 McGonnell IM, Green CR, Tickle C, et al. Connexin43 gap junction protein plays an essential role in morphogenesis of the embryonic chick face. Dev Dyn 2001;222:420-38

41 Griffith CM, Hay E, eds. Epithelial-mesenchymal transformation during palatal fusion: carboxyfluorescein traces cells at light and electron microscopic levels. Development 1992;116:1087-99.

42 Shuler CF, Halpern DE, Guo Y, et al. Medial edge epithelium fate traced by cell lineage analysis during epithelial-mesenchymal transformation in vivo. Dev Biol 1992;154:318-30.

43 Nishii K, Kumai M, Shibata Y. Regulation of the epithelial-mesenchymal transformation through gap junction channels in heart development. Trends Cardiovasc Med 2001;11:213-8.

44 Inai T, Nakamura K, Kurisu K, et al. Immunohistochemical localization of connexin 43 in the enamel organ of the rat upper incisor during ameloblast development. Arch Histol Cytol 1997;60:297-306.

45 Murakami S, Muramatsu T, Shimono M. Expression and localization of connexin 43 in rat incisor odontoblasts. Anat Embryol 2001 ;203:367-74.

46 Ibuki N, Yamaoka Y, Sawa Y, et al. Different expressions of connexin 43 and 32 in the fibroblasts of human dental pulp. Tissue Cell 2002;34:170-6

47 Ruangvoravat CP, Lo CW. Connexin 43 expression in the mouse embryo: localization of transcripts within developmentally significant domains. Dev Dyn 1992; 194:261-81.

48 van der Heyden MA, Roeleveld L, Peterson J, et al. Connexin 43 expression during Xenopus development. Mech Dev 2001;108:217-20.

49 Becker DL, Bonness V, Catsicas $M$, et al. Changing patterns of ganglion cell coupling and connexin expression during chick retinal development. J Neurobiol 2002;52:280-93

50 Meyer RA, Cohen MF, Recalde S, et al. Developmental regulation and asymmetric expression of the gene encoding $\mathrm{C} \times 43$ gap junctions in the mouse limb bud. Dev Genet 1997;21:290-300.

\section{$\mathrm{ECHO}$}

\section{A new PHOX2B polymorphism is associated with Hirschsprung's disease}

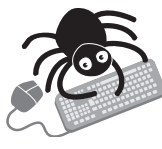

Please visit the Journal of Medical Genetics website [www. jmedgenet. com] for a link to the full text of this article. nravelling the complexities of Hirschsprung's disease (HSCR) is a step closer, with the first association study of $\mathrm{PHOX} 2 \mathrm{~B}$ as a candidate gene in Chinese people. The disease is polygenic so gene interactions may occur-but first, the susceptibility genes must be identified.

An $\mathrm{A} \rightarrow \mathrm{G}_{1364}$ substitution in intron 2-one of three new PHOX2B polymorphisms founddiffered significantly in genotype frequency between patient and control groups and controls and almost all of another patient group with the disease but no RET mutation. Significant linkage disequilibrium occurred between this and a $\mathrm{DEL}_{2609}$ deletion of five alanine residues at codons 254-258, but whether directly or by gene interaction is unclear, say the authors. There were no sex differences in genotype frequencies of each of the three polymorphisms, and each polymorphism in all groups was in Hardy-Weinberg equilibrium.

DNA amplification and sequencing of the whole $P H O X 2 B$ gene and its flanking regions were performed in 9l patients with HSCR in Hong Kong, 71 ethnically matched Chinese controls, and 75 patients with HSCR but no RET mutation.

Ganglion cells are absent in the nerve plexus of the lower gut in HSCR. RET expression and HSCR genes coding for proteins in two signalling pathways-RET and endothelin receptor B pathways-account for only some cases. Neurone development also depends on a transcription factor encoded by the.PHOX2B gene. Mice with the homozygous mutation have no enteric ganglia and do not express RET, suggesting that $P H O X 2 B$ regulation of RET determines nerve development- the hypothesis tested in the current study.

A Gut 2003;52:563-567. 